\title{
Symptom clusters among cancer survivors: what can machine learning techniques tell us?
}

Koen I. Neijenhuijs ${ }^{1,2}$, Carel F. W. Peeters ${ }^{3,4}$, Henk van Weert ${ }^{5}$, Pim Cuijpers ${ }^{1}$ and Irma Verdonck-de Leeuw ${\text { I, } 2,6^{*}}^{*}$

\begin{abstract}
Purpose: Knowledge regarding symptom clusters may inform targeted interventions. The current study investigated symptom clusters among cancer survivors, using machine learning techniques on a large data set.

Methods: Data consisted of self-reports of cancer survivors who used a fully automated online application 'Oncokompas' that supports them in their self-management. This is done by 1) monitoring their symptoms through patient reported outcome measures (PROMs); and 2) providing a personalized overview of supportive care options tailored to their scores, aiming to reduce symptom burden and improve health-related quality of life. In the present study, data on 26 generic symptoms (physical and psychosocial) were used. Results of the PROM of each symptom are presented to the user as a no well-being risk, moderate well-being risk, or high well-being risk score. Data of 1032 cancer survivors were analysed using Hierarchical Density-Based Spatial Clustering of Applications with Noise (HDBSCAN) on high risk scores and moderate-to-high risk scores separately.
\end{abstract}

Results: When analyzing the high risk scores, seven clusters were extracted: one main cluster which contained most frequently occurring physical and psychosocial symptoms, and six subclusters with different combinations of these symptoms. When analyzing moderate-to-high risk scores, three clusters were extracted: two main clusters were identified, which separated physical symptoms (and their consequences) and psycho-social symptoms, and one subcluster with only body weight issues.

Conclusion: There appears to be an inherent difference on the co-occurrence of symptoms dependent on symptom severity. Among survivors with high risk scores, the data showed a clustering of more connections between physical and psycho-social symptoms in separate subclusters. Among survivors with moderate-to-high risk scores, we observed less connections in the clustering between physical and psycho-social symptoms.

Keywords: Cancer, Oncology, Symptom clusters, Machine learning

\footnotetext{
* Correspondence: im.verdonck@amsterdamumc.nl

'Department of Clinical, Vrije Universiteit Amsterdam, Neuro- and

Developmental Psychology, Amsterdam Public Health Research Institute, Van

der Boechorststraat 1, 1081, BT, Amsterdam, The Netherlands

${ }^{2}$ Amsterdam UMC, Cancer Center Amsterdam, Amsterdam, The Netherlands

Full list of author information is available at the end of the article
}

(C) The Author(s). 2021 Open Access This article is licensed under a Creative Commons Attribution 4.0 International License, which permits use, sharing, adaptation, distribution and reproduction in any medium or format, as long as you give appropriate credit to the original author(s) and the source, provide a link to the Creative Commons licence, and indicate if changes were made. The images or other third party material in this article are included in the article's Creative Commons licence, unless indicated otherwise in a credit line to the material. If material is not included in the article's Creative Commons licence and your intended use is not permitted by statutory regulation or exceeds the permitted use, you will need to obtain permission directly from the copyright holder. To view a copy of this licence, visit http://creativecommons.org/licenses/by/4.0/ The Creative Commons Public Domain Dedication waiver (http://creativecommons.org/publicdomain/zero/1.0/) applies to the data made available in this article, unless otherwise stated in a credit line to the data. 


\section{Introduction}

Cancer survivors experience a myriad of symptoms rooted in physiology caused by the disease itself or caused by the treatment thereof [1]. Problems in the psychosocial domain are also prevalent [2-4]. Many of these symptoms and problems may co-occur and are likely interrelated. For example, sleep problems have been identified as both a risk factor and as a symptom of depression in both cancer [5] and non-cancer populations [6]. In cancer patients, subjective cognitive functioning has been associated with depression, anxiety, and fatigue [7]. Furthermore, problems with sexual health have been related to body image issues, depression, and anxiety [8]. Fatigue has been associated with pain, sleep issues, and depression; and nausea with vomiting [9].

Such interrelated symptoms are referred to as symptom clusters, and knowledge regarding such symptom clusters may inform targeted interventions [10]. Some studies have set out to empirically determine symptom clusters using various types of cluster analyses. In 2011, a systematic review identified 47 studies that statistically investigated symptom clusters in cancer patients [9]. A number of symptom clusters repeatedly showed up: (i) a fatigue-depression-pain cluster, (ii) a nausea-vomiting cluster, and (iii) a depression-anxiety-insomnia cluster. However, the authors note that these (and other) clusters seem heavily influenced by the population studied (tumor type, cancer stage, treatment modality and treatment intent), symptom assessment method, and statistical method used. Also, many of these studies were limited in scope in terms of sample size, number of symptoms investigated, or the type of analysis that was used.

Another systematic review was performed for studies up to 2016 which focused on cancer patients receiving primary or adjuvant chemotherapy [11]. Nineteen studies were included, and a few consistently appearing symptom clusters were identified: (i) a nausea-vomiting cluster, (ii) a psychological symptom cluster, and (iii) a "sickness behavior" (pain-fatigue-insomnia-lack of appetite) cluster. Noteworthy is that the individual symptoms in each of these clusters were not necessarily consistent between studies.

In 2015 an international expert panel regarding "Advancing Symptom Science Through Symptom Cluster Research" was formed [10]. This panel was subdivided into five groups: (i) defining characteristics of symptom clusters, (ii) identification of priority symptom clusters and underlying mechanisms, (iii) measurement of symptom clusters, (iv) targeted interventions for symptom clusters, and (v) new analytic strategies for symptom cluster research. In line with the aforementioned review [9], the first expert group concluded that there is little consistency in the number and types of symptom clusters identified in cancer patients/survivors. This expert group defined a number of directions for future research in defining symptom clusters. In particular, they stated a need for "the establishment of a common conceptual framework and approach for the evaluation of measurement of symptom clusters" and "the evaluation of the potential to use large data sets and electronic health records to evaluate symptom clusters". The fifth expert group also defined some directions for future research, one of which was to "apply new analytic techniques to symptom cluster research". The international expert panel noted that the investigation of symptom clusters may result in the identification of causal relationships between symptoms, possibly informing targeted interventions on symptoms with a causal force [10].

Currently, we are in limbo of the explorative phase regarding symptom cluster research, where universal symptom clusters require identification. The inconsistencies in symptom clusters as identified by the aforementioned systematic reviews $[9,11]$ and corroborated by the international panel [10] are preventing the field of symptom cluster research to move into confirmatory research. The current study hopes to contribute to identifying symptom clusters by using a robust and innovative explorative analysis method.

Previous symptom cluster research is predominated by methods focused on latent variable (mixture) modeling. This method classifies individuals into unobserved clusters with (more) homogenous patterns within the clusters. Models based on homogeneity have the drawback that if most individuals are relatively alike, very large clusters will be identified. The previous literature $[9,11]$ indicates that most cancer patients are all very sick. This often creates a "general sickness" cluster. However, to identify symptom clusters we strive to identify the subclusters within such a larger cluster. Density-based clustering methods are designed to identify clusters of varying densities. As such, these methods are particularly useful in separating smaller subclusters (with higher densities) from larger clusters (with lower densities). A recent development in the field of density-based clustering methods is Hierarchical Density-Based Spatial Clustering of Applications with Noise (HDBSCAN) [12].

One further limitation to previous symptom cluster research is the limited breadth of symptoms studied [9]. Research has shown that cancer patients and survivors suffer a broad range of symptoms and other health issues [13-17]. These other health issues include lifestyle behaviors such as smoking or psychosocial constructs such as heightened stress, that do not fit into a semantic definition of 'symptoms'. Nevertheless, they are likely interrelated and contribute to the disease profile of 
patients and survivors. As such, the current study sought to include these constructs, by clustering both symptoms and health determinants.

The aim of this study is to investigate symptom clusters among cancer patients and survivors, by analyzing a large dataset of a broad range of self-reported symptoms using HDBSCAN [12]. The results of the present study will contribute to the establishment of a conceptual framework and an approach for the evaluation of symptom and health determinants cluster measurements.

\section{Methods}

\section{Study population}

The study sample consisted of users of the eHealth application Oncokompas [13]. These users are Dutch cancer patients (currently undergoing treatment) or cancer survivors (treatment has ended) who are/were treated with curative intent. The application is available for patients and survivors through referral of their main healthcare provider. In total, data of 1032 users were included, who consented to the use of their data for research purposes. Of these users, 715 users of Oncokompas were referred by a healthcare provider in routine care, 191 cancer survivors were invited to participate in a randomized controlled trial (RCT) investigating the efficacy of Oncokompas [13], 72 colon cancer survivors were invited to participate in a multi-centre RCT [14], and 54 breast cancer survivors were invited to participate in a pilot on the feasibility of Oncokompas [15].

\section{Materials}

Oncokompas is a fully automated online application that supports cancer survivors in their self-management by 1) monitoring their health-related quality of life (HRQOL) and (cancer-generic and tumour-specific) symptoms and health determinants; and 2) providing tailored feedback on their scores with a personalized overview of supportive care options, with the aim to reduce symptom burden and improve HRQOL [13]. Oncokompas covers a total of 46 topics on five generic domains applicable for all cancer survivors: physical, psychological, and social HRQOL, healthy lifestyle, and existential topics; and 29 tumour-specific topics for survivors of breast cancer, colorectal cancer, head and neck cancer, and lymphoma. Users can choose which topics they wish to fill in. In the current study, only the generic topics were used. Oncokompas consists of three components: 'Measure', 'Learn', and 'Act'. For the current study, only the Measure component is of interest, the Learn and Act component are detailed elsewhere [13]. In the Measure component, users can independently complete patient reported outcome measures (PROMs) targeting the selected topic(s). On each of the selected topics, the user receives a green (no well-being risk), orange (moderate well-being risk), or red (high well-being risk) outcome. The current study focuses on 26 of the 46 generic topics, as these 26 topics represent physical or psycho-social symptoms and health determinants that often occur based on literature [16]. Table 1 details the symptoms and health determinants, and PROMs that were used in the analysis, as well as the possible color outcomes on each PROM. Each symptom and health determinant consists of one or multiple PROMs, which were selected by the project team in collaboration with expert teams and based on Dutch practical guidelines (from the Netherlands Comprehensive Cancer Organisation) and literature searches [17].

\section{Data analysis}

Data of cancer survivors who used Oncokompas up to April 29th 2019 were used. Users can fill in Oncokompas more than once. Unfortunately, not enough users filled in Oncokompas more than once to be able to analyze trends over time. To remove within-user variance, when users had filled in Oncokompas more than once, one random time point was selected of the user for the current study. Sensitivity analyses were run by repeating the analysis a number of times using different random seeds for row selection. The results did not differ regarding the main results, and will not further be discussed. All analyses were run in R version 3.5.3 [18], or in Python version 3.7.1 [19]. Two types of analyses were used: network analysis and cluster analysis.

For the cluster analyses, HDBSCAN [12] was performed using the hdbscan library in Python [20]. HDBSCAN separates a dataset into clusters of high and low density. HDBSCAN is an extension of the DBSCAN clustering algorithm [21], where HDBSCAN is capable of identifying clusters of varying densities and is more robust to parameter selection [20]. This makes the HDBSCAN algorithm particularly useful in separating smaller subclusters (with higher densities) from larger clusters (with lower densities). Data points that do not fit into any of the identified clusters are labeled as noise by the algorithm. The Jaccard distance metric was used due to the categorical nature of our measurement of a symptoms and health determinants. The minimum points required to form a cluster (minimum cluster size) was set to 26 (number of modules). Because we were interested in subclusters of symptoms and health determinants, the minimum sample was set to 1 , and leaf clustering was used for cluster selection. These parameters prioritize the extraction of multiple smaller rather than larger clusters. Due to the explorative nature of the analysis we did not perform hyperparameter tuning to find the parameters that lead to the least amount of noise. The parameters resulting from hyperparameter 
Table 1 Overview of Oncokompas topics

\begin{tabular}{|c|c|c|}
\hline Topics & PROM & Possible scores \\
\hline Contact with doctor & EORTC IN-PATSAT32 & Green; Orange \\
\hline Dedication to work & Visual Analogue Scale & Green; Orange \\
\hline Smoking & Oncokompas expert-based questionnaire & Green; Orange; Red \\
\hline Alcohol use & Alcohol 5-shot & Green; Orange; Red \\
\hline Relaxation & Perceived Stress Scale & Green; Orange; Red \\
\hline Physical activity & Oncokompas expert-based questionnaire & Green; Orange \\
\hline Body weight & BMI \& Short Nutritional Assessment Questionnaire & Green; Orange; Red \\
\hline Physical limitations daily life & Patient Specifieke Klachtenlijst (Dutch-specific) & Green; Orange; Red \\
\hline Insomnia & Insomnia Severity Index & Green; Orange; Red \\
\hline Fatigue & Numeric Rating Scale & Green; Orange; Red \\
\hline Pain & Numeric Rating Scale & Green; Orange; Red \\
\hline Constipation & Numeric Rating Scale & Green; Orange; Red \\
\hline Diarrhea & Numeric Rating Scale & Green; Orange; Red \\
\hline Lack of appetite & Numeric Rating Scale & Green; Orange; Red \\
\hline Nausea or vomiting & Numeric Rating Scale & Green; Orange; Red \\
\hline Shortness of breath & Numeric Rating Scale & Green; Orange; Red \\
\hline Hearing problems & Caron hearing questionnaire & Green; Orange; Red \\
\hline Tinnitus & Oncokompas expert-based questionnaire & Green; Orange; Red \\
\hline Psychological complaints & Hospital Anxiety and Depression Scale & Green; Orange; Red \\
\hline Memory / concentration & SF-36 'cognitive functioning' & Green; Orange; Red \\
\hline Social life & De Jong-Gierveld Loneliness Scale & Green; Orange; Red \\
\hline Financial problems & EORTC QLQ-C30 'financial problems' & Green; Orange; Red \\
\hline Intimacy and sexuality & Female Sexual Function Index (women) / International Index of Erectile Function (men) & Green; Orange; Red \\
\hline Body image & Body Image Scale & Green; Orange; Red \\
\hline Relationship with partner & Dyadic Adjustment Scale Short Form & Green; Orange; Red \\
\hline Relationship with children & Vragenlijst Gezinskernmerken Short Form (Dutch-specific) & Green; Orange; Red \\
\hline
\end{tabular}

tuning may not be able to extract the subclusters with higher densities that we are interested in, instead extracting the larger clusters with lower densities.

The network analyses were performed using the tidygraph [22] and ggraph [23] packages in R. The network graphs were nondirectional, and edges were calculated as the raw number of connections between nodes (i.e. the occurrence of symptom-pairs among the same patient). Weighted degree centrality was calculated using the edges as weights.

The choice was made to perform the clustering based on the presence of a symptom or health determinant, instead of a continuous score of severity. Methodologically, clustering based on a dichotomous presence of a symptom or health determinant results in clusters that are more easily interpretable (i.e. "all the patients in this cluster have this symptom") than clusters based on symptom severity (i.e. "these patients share a similar range of symptom severity"). The benefit of a more easily interpretable cluster is two-fold. Firstly, it will hopefully lead to more easily replicable symptom and health determinant clusters in follow-up confirmatory research. Secondly, the knowledge regarding identified symptom and health determinant clusters it will hopefully be more useful in practice. We theorize it is easier for clinicians to relate the presence of symptoms and health-related issues to one another, than it is to relate the severity of such symptoms and health-related issues to one another. For example, it appears easier to identify the probability of insomnia being present when a patient has been diagnosed with depression, rather than the probability of insomnia being present based on a severity scale of depression.

One analysis was run on only high risk (red) scores as the definition of a symptom or health determinants being present, as these scores are based on cut-off scores with most empirical evidence. Three symptoms on which a high risk scores was not possible (see Table 1) were excluded for this analysis. While severity was not chosen as the operationalization of a symptom or health 
determinant, it may be an influence on how symptoms and health determinants cluster together [9]. Consequently, a second analysis was run on moderate-to-high risk (orange and red) scores as the definition of a symptom or health determinant being present, which also included the previously excluded symptoms and health determinants.

\section{Results}

\section{Patient characteristics}

Table 2 shows the patient characteristics. The mean age was 61.5 years (range 25-88), the majority was female (68\%), approximately half was treated for breast cancer (49\%), most had completed treatment (60\%), and most patients were treated with surgery (79\%).

\section{High risk score analysis}

In the high risk score analysis, seven clusters were extracted. A total of 393 data points were deemed noise, which amounted to $19.31 \%$ of the data, which is a nonnegligible amount.

The cluster profiles are presented in Table 3. The cell numbers represent how many patients with a certain symptom or health determinant were present in any given cluster. The largest cluster (cluster 7), represents a "general sickness cluster", encompassing patients who suffer from most symptoms and health determinants that were present in the data set. Next are two clusters that represent patients who experienced one symptom almost exclusively: a psychological complaint cluster (cluster 1), and a physical limitations cluster (cluster 2). Cluster 3 represents patients who mainly experienced symptoms and health determinants regarding body weight, alcohol use, and social life, while cluster 4 represents patients who mainly experience symptoms and health determinants regarding physical limitations, intimacy/sexuality, and body weight. Cluster 5 is the second-largest cluster with regard to number of symptoms and health determinants, and represents patients who experienced psychological symptoms and health determinants, and various physical symptoms. Lastly, cluster 6 represents patients who experienced psychological complaints, problems with relaxation, and social life.

Figure 1 shows the network plot of the main analysis. The plot shows both the main cluster of each symptom or health determinant (the cluster in which the symptom or health determinant is most frequent), as well as the subcluster of each symptom or health determinant (the cluster in which the symptom or health determinant is second-most frequent, with a minimum frequency of 5). Psychological complaints and physical limitations have the highest weighted degree centrality and are connected to nearly all symptoms and health determinants. The
Table 2 Descriptive statistics

\begin{tabular}{|c|c|c|c|c|c|}
\hline & Mean & SD & & $\mathbf{N}$ & $\%$ \\
\hline Age & 60.52 & 11.31 & & & \\
\hline \multirow[t]{2}{*}{ Gender } & & & Female & 701 & $67.99 \%$ \\
\hline & & & Male & 330 & $32.01 \%$ \\
\hline \multirow[t]{7}{*}{ Education } & & & Elementary school & 24 & $2.33 \%$ \\
\hline & & & High school & 164 & $15.91 \%$ \\
\hline & & & Vocational education & 590 & $57.23 \%$ \\
\hline & & & College & 115 & $11.15 \%$ \\
\hline & & & University & 107 & $10.38 \%$ \\
\hline & & & Post-doctoral & 23 & $2.23 \%$ \\
\hline & & & Other & 8 & $0.78 \%$ \\
\hline \multirow[t]{16}{*}{ Cancer type } & & & Breast cancer & 504 & $48.88 \%$ \\
\hline & & & Colon cancer & 182 & $17.65 \%$ \\
\hline & & & Lymphoma & 73 & $7.08 \%$ \\
\hline & & & Head and neck cancer & 60 & $5.82 \%$ \\
\hline & & & Rectal cancer & 40 & $3.88 \%$ \\
\hline & & & Other & 39 & $3.78 \%$ \\
\hline & & & Lung cancer & 31 & $3.01 \%$ \\
\hline & & & Prostate cancer & 29 & $2.81 \%$ \\
\hline & & & Gynecologic cancer & 20 & $1.94 \%$ \\
\hline & & & Bladder or kidney cancer & 17 & $1.65 \%$ \\
\hline & & & Skin cancer & 11 & $1.07 \%$ \\
\hline & & & Blood cancer & 9 & $0.87 \%$ \\
\hline & & & Esophageal cancer & 5 & $0.48 \%$ \\
\hline & & & Brain cancer & 4 & $0.39 \%$ \\
\hline & & & Pancreatic or liver cancer & 4 & $0.39 \%$ \\
\hline & & & Stomach cancer & 3 & $0.29 \%$ \\
\hline \multirow[t]{5}{*}{ Treatment status } & & & Treatment completed & 614 & $59.55 \%$ \\
\hline & & & Currently being treated & 172 & $16.68 \%$ \\
\hline & & & Not yet treated & 94 & $9.12 \%$ \\
\hline & & & Unknown & 80 & $7.76 \%$ \\
\hline & & & No treatment & 71 & $6.89 \%$ \\
\hline \multirow[t]{9}{*}{ Treatment type } & & & Surgical & 743 & $79.04 \%$ \\
\hline & & & Chemotherapy & 103 & $10.96 \%$ \\
\hline & & & Radiation & 38 & $4.04 \%$ \\
\hline & & & Chemoradiation & 24 & $2.55 \%$ \\
\hline & & & Other & 10 & $1.06 \%$ \\
\hline & & & Hormone therapy & 7 & $0.74 \%$ \\
\hline & & & Wait-and-see & 7 & $0.74 \%$ \\
\hline & & & Immunotherapy & 4 & $0.43 \%$ \\
\hline & & & Unknown & 4 & $0.43 \%$ \\
\hline
\end{tabular}

intra-cluster connections range from large (mostly connections originating from psychological complaints or physical limitations), to moderate (most intra-cluster connections), to small (mostly connections originating 
Table 3 High risk cluster profiles

\begin{tabular}{|c|c|c|c|c|c|c|c|c|}
\hline Symptom/Health Determinant & Noise & Cluster 1 & Cluster 2 & Cluster 3 & Cluster 4 & Cluster 5 & Cluster 6 & Cluster 7 \\
\hline Psychological complaints & 225 & 65 & & 1 & 6 & 36 & 30 & 52 \\
\hline Fatigue & 114 & & & 1 & 6 & 21 & 1 & 48 \\
\hline Physical limitations daily life & 100 & & 27 & & 18 & 33 & 5 & 46 \\
\hline Memory / concentration & 53 & & & & & 1 & 3 & 26 \\
\hline Relaxation & 123 & & & & & 1 & 31 & 25 \\
\hline Social life & 82 & & & 12 & & 9 & 29 & 24 \\
\hline Insomnia & 66 & & & 1 & 1 & 3 & 2 & 21 \\
\hline Pain & 64 & & & 1 & & 10 & 1 & 19 \\
\hline Intimacy and sexuality & 74 & & & 1 & 15 & 11 & & 15 \\
\hline Hearing problems & 107 & & & & 4 & 26 & 1 & 15 \\
\hline Shortness of breath & 47 & & & & 2 & 15 & 1 & 9 \\
\hline Body weight & 35 & & & 22 & 14 & 3 & & 8 \\
\hline Constipation & 25 & & & & & & 3 & 3 \\
\hline Smoking & 41 & & & 3 & & 6 & 1 & 2 \\
\hline Diarrhea & 33 & & & & & & & 2 \\
\hline Body image & 15 & 3 & & & & & & 1 \\
\hline Relationship with partner & 21 & & & & & 1 & 4 & 1 \\
\hline Alcohol use & 22 & & & 12 & 2 & & 2 & \\
\hline Lack of appetite & 23 & & & & & & & \\
\hline Relationship with children & 19 & & & & & & & \\
\hline Nausea or vomiting & 18 & & & & & & & \\
\hline Financial matters & 17 & & & & & & & \\
\hline Tinnitus & 6 & & & & & & & \\
\hline
\end{tabular}

Note: The cell numbers represent the number of patients with a certain symptom / health determinant in any given cluster. Noise indicates data points that do not fit into any of the identified clusters

from fringe symptoms or health determinants with less neighbors).

\section{Moderate-to-high risk score analysis}

In the moderate-to-high risk score analysis, three clusters were extracted. A total of 579 data points were deemed as noise, which amounted to $39.25 \%$ of the data, which is a high amount.

The cluster profiles are presented in Table 4. One small cluster was extracted with patients who only experienced health determinants regarding body weight (Cluster 1). Two large clusters emerged: a lifestyle and psychosocial cluster (cluster 2), and a physical symptoms cluster (Cluster 3).

Figure 2 shows the network plot of the sensitivity analysis. The plot does not show the body weight cluster, as this health determinant was strongly incorporated into the psychosocial and lifestyle cluster. Psychological complaints, physical limitations, physical activity, relaxation, and fatigue have the highest weighted degree centrality and are connected to nearly all symptoms and health determinants. The intra-cluster connections range from large (mostly connections originating from the symptoms and health determinants with high weighted degree centrality), to moderate (most intra-cluster connections), to small (mostly connections originating from fringe symptoms and health determinants with less neighbors). This network analysis shows more inter-cluster connections than the main analysis. Strong connections exist between psychological complaints (cluster 2) and fatigue (cluster 3), and physical limitations (cluster 3). Moderate connections exist between psychological complaints (cluster 2) and intimacy/sexuality (cluster 3), pain (cluster 3 ), and insomnia (cluster 3 ). Moderate connections exist between physical limitations (cluster 3) and social life (cluster 2), physical activity (cluster 2), relaxation (cluster 2), and social life (cluster 2).

\section{Discussion}

In this explorative study we used HDBSCAN to extract symptom and health determinant clusters based on scores on PROMs of cancer patients/survivor who used the eHealth application Oncokompas. When analyzing the high risk scores of patients, different clusters 


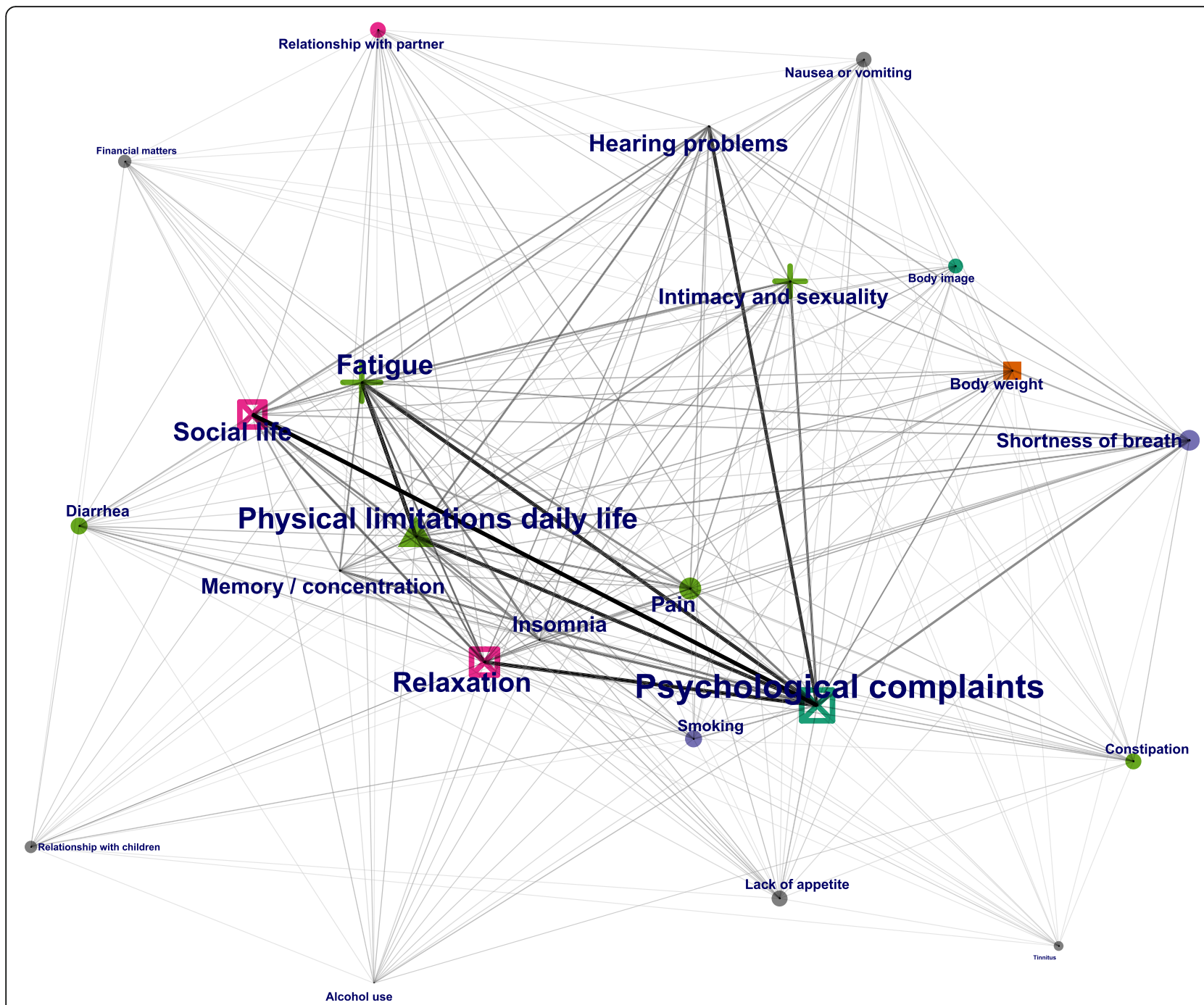

Fig. 1 Network plot high risk score analysis. Every point in the network plot represents a symptom or health determinant as defined in Table 1. The size of the points represents the amount of connections it has with other symptoms and health determinants (weighted degree centrality). The thickness of the lines represents the amount of connections between those two specific symptoms or health determinants. Symptoms and health determinants are colored based on their main cluster (the cluster in which the symptom or health determinant is most frequent), and shapes are based on their subcluster (the cluster in which the symptom or health determinant is second-most frequent, with a minimum frequency of 5)

appeared compared to the analysis of moderate-to-high risk scores. When analyzing patients showing high-risk scores, we found one overarching cluster containing most symptoms and health determinants measured, as well as six subclusters. When analyzing patients showing moderate-to-high risk scores, we found two overarching clusters: one representing psychosocial symptoms and health determinants, and one representing physical symptoms and their health consequences.

This study was explorative in nature, and while the symptoms and health determinants used in the current study do not entirely line up with all symptoms reported on by previous research [5, 7-9, 11], it is of interest to see whether symptom and health determinant clusters identified in our dataset line up with the symptom clusters previously identified. First, a fatigue-depression-pain cluster $[9,11]$ has been reported by previous studies. We found fatigue, psychological complaints, and pain to be clustered together in the "general sickness" cluster as well as in the "physical symptoms and consequences" cluster in the high risk score analysis. And while fatigue and pain clustered in the "physical symptoms and consequences" cluster in the moderate-to-high risk score analysis, they were not clustered with psychological complaints.

Second, previous literature showed evidence for a depression-anxiety-insomnia cluster [5, 9]. In the present 
Table 4 Moderate-to-high risk cluster profiles

\begin{tabular}{|c|c|c|c|c|}
\hline Symptom/Health Determinants & Noise & Cluster 1 & Cluster 2 & Cluster 3 \\
\hline Physical activity & 257 & & 175 & 130 \\
\hline Relaxation & 105 & & 88 & 67 \\
\hline Insomnia & 99 & & 5 & 67 \\
\hline Contact with doctor & 75 & & 46 & 66 \\
\hline Shortness of breath & 79 & & 1 & 65 \\
\hline Physical limitations daily life & 100 & & 2 & 51 \\
\hline Fatigue & 136 & & 3 & 50 \\
\hline Social life & 58 & & 57 & 43 \\
\hline Alcohol use & 147 & & 98 & 39 \\
\hline Tinnitus & 85 & & 3 & 39 \\
\hline Pain & 59 & & & 38 \\
\hline Financial matters & 71 & & 52 & 36 \\
\hline Lack of appetite & 26 & & & 25 \\
\hline Intimacy and sexuality & 58 & & 2 & 24 \\
\hline Relationship with partner & 39 & & 19 & 18 \\
\hline Psychological complaints & 96 & & 74 & 16 \\
\hline Constipation & 26 & & 1 & 16 \\
\hline Nausea or vomiting & 16 & & & 16 \\
\hline Hearing problems & 26 & & 3 & 11 \\
\hline Memory / concentration & 13 & & & 11 \\
\hline Body weight & 62 & 18 & 123 & 10 \\
\hline Relationship with children & 36 & & 32 & 10 \\
\hline Body image & 16 & & & 10 \\
\hline Dedication to work & 28 & & 1 & 8 \\
\hline Diarrhea & 5 & & & 4 \\
\hline Smoking & 15 & & 7 & 2 \\
\hline
\end{tabular}

Note: The cell numbers represent the number of patients with a certain symptom or health determinant in any given cluster. Noise indicates data points that do not fit into any of the identified clusters)

study, insomnia and psychological complaints were clustered together in the "general sickness" cluster, and showed a moderate connection in the network analysis (weight $=76$ ) in the high risk score analysis. In the moderate-to-high risk score analysis insomnia and psychological complaints were not clustered together, but did show a strong connection in the network analysis (weight $=219$ ).

Third, a psychological symptom cluster was found in multiple previous studies $[9,11]$. In the high risk score analysis we found both a "psychological complaints" and "psychosocial" cluster. However, in the moderate-to-high risk score analysis we found a broader psychosocial and lifestyle cluster.

Fourth, a pain-fatigue-insomnia-lack of appetite clusters was reported [11]. In the high risk score analysis, pain, fatigue, and sleeping issues clustered together in the "general sickness" cluster; and pain and fatigue clustered in the "physical symptoms and consequences" cluster. Meanwhile, in the moderate-to-high risk score analysis, pain, fatigue, sleeping issues, and lack of appetite clustered in the "physical symptoms and consequences" cluster.

Fifth, a specific association between cognitive functioning and psychological distress was found in previous literature [7]. In our high risk score analysis, memory/ concentration and psychological complaints were clustered together in the "general sickness" cluster, and showed a moderate connection in the network analysis (weight $=66$ ); while in the moderate-to-high risk score analysis memory/concentration and psychological complaints were not clustered together, but did show a moderate connection in the network analysis (weight $=98$ ).

Sixth, literature showed a specific association between memory/concentration and fatigue [7]. In our high risk score analysis, memory/concentration and fatigue clustered in the "general sickness" cluster, and showed a small to moderate connection in the network analysis 


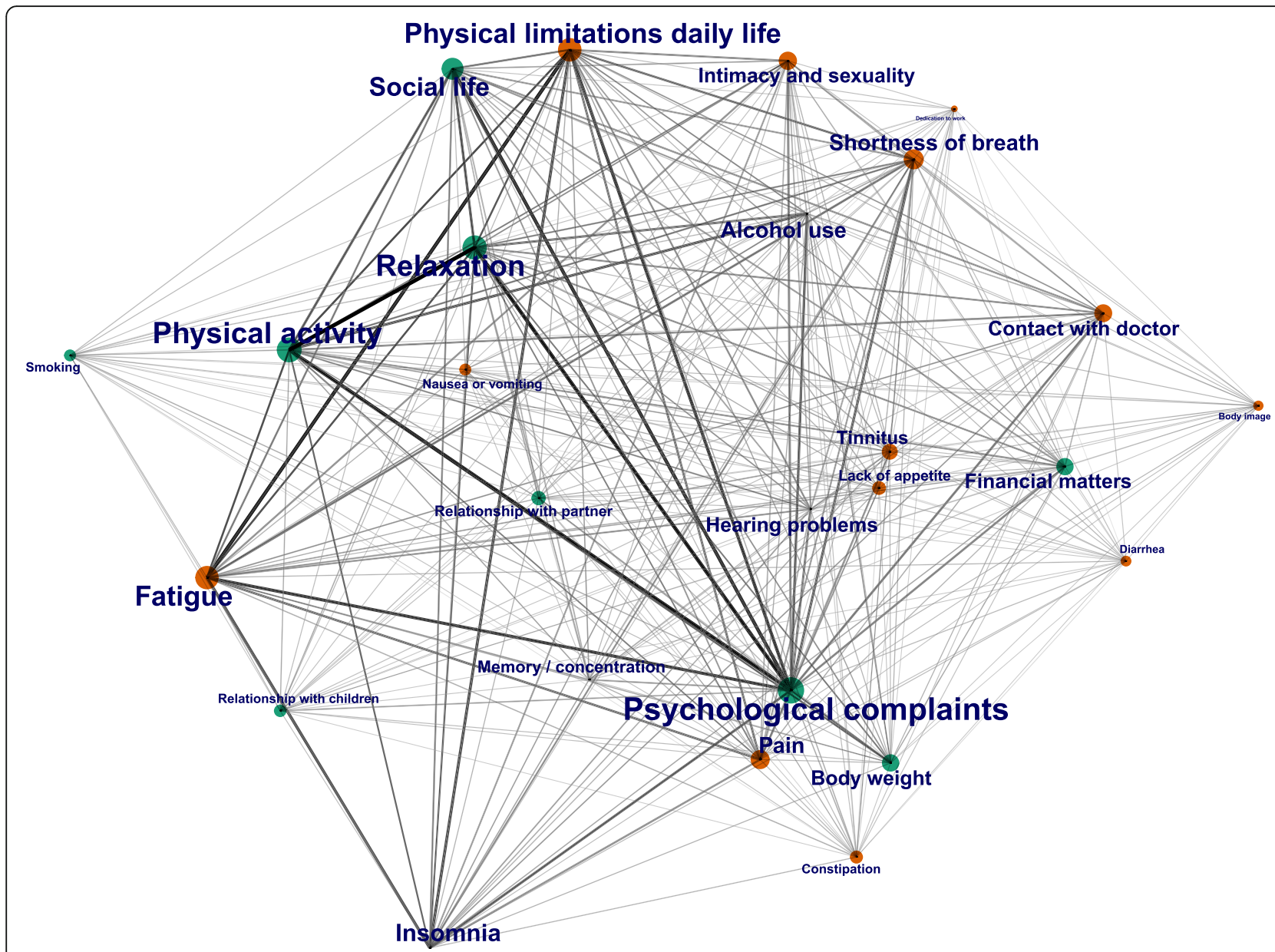

Fig. 2 Network plot moderate-to-high risk score analysis. Every point in the network plot represents a symptom/health determinant as defined in Table 1. The size of the points represents the amount of connections it has with other symptoms and health determinants (weighted degree centrality). The thickness of the lines represents the amount of connections between those two specific symptoms or health determinants. Symptoms and health determinants are colored based on their main cluster (the cluster in which the symptom or health determinant is most frequent)

(weight $=54$ ). In the moderate-to-high risk score analysis, memory/concentration and fatigue clustered in the "physical symptoms and consequences" cluster, and showed a moderate connection in the network analysis ( weight $=93$ ).

Seventh, a specific association between sexual problems and body image has been reported [8]. Intimacy/ sexuality and body image were not clustered together in our high risk score analysis, as body image was not part of any cluster, and showed a very weak connection in the network analysis (weight $=5$ ). In the moderate-to-high risk score analysis, intimacy/sexuality and body image clustered in the "physical symptoms and consequences" cluster, but showed a weak connection in the network analysis (weight $=22$ ). These results may be explained by the fact that we have very few patients in the main data set with body image problems.
Eight and last, a specific association between sexual problems and psychological distress was found previously [8]. In our high risk score analysis, intimacy/sexuality and psychological complaints were clustered together in the "general sickness" cluster as well as in the "physical symptoms and consequences" cluster, and showed a moderate connection in the network analysis (weight $=73$ ). In the moderate-to-high risk score analysis, intimacy/sexuality and psychological complaints were not clustered together, but did show a strong connection in the network analysis (weight $=159$ ).

These results show that many of the previously reported (sub) clusters were found in our high risk score analysis, but not in the moderate-to-high risk score analysis. There may be an inherent difference on the cooccurence of symptoms and health determinants dependent on severity. For patients with higher severity, we observed more connections between the physical and 
psycho-social symptoms and health determinants, compared to patients with lower severity. This provokes the question of causality: do patients with higher severity of physical issues develop higher severity of psycho-social issues, vice versa, or are both higher severities developed in tandem due to a third causal force? The statistical methods we used are associative, intending to identify clusters of co-occuring symptoms and health determinants which do not necessarily share the same etiology [10]. As such, we cannot offer an answer to the question of causality. But future research could use methodology more suited for such investigations.

It has been suggested that cluster symptoms are not the same across different cancer diagnoses [11]. The current study did not perform subgroup analyses between cancer diagnoses, as the corresponding sample sizes would not have been sufficient for all diagnoses. In future research, after Oncokompas has attracted more users of differing diagnoses, such subgroup cluster analyses may provide further insights into this possibility.

While many studies researching symptom clusters predominantly use variations of exploratory and confirmatory factor analyses $[9,11]$, innovation in methodology is starting to gain traction. The current study made use of an explorative machine learning algorithm and a rather simple method of network analysis. Other studies innovated through the use of new and more complex network analysis methods: Pairwise Markov Random Field (PMRF) [24] and concordance networks [25]. These methods create more robust networks of symptoms, but the estimation of clusters is inherently different than through the use of a specific cluster algorithm. The clusters (i.e. communities in network terminology) are defined through the WalkTrap [24] or random walk [25] algorithms which identifies clusters of network nodes (i.e. symptoms) that are highly connected (i.e. higher valued edges). To put it more simply: while the current study identifies symptom and health determinant clusters based on the density of co-occuring symptoms, the network methodology identifies symptom clusters based on the strength of network edges.

As these are two distinctly different ways of defining what a cluster entails, it is of interest to see whether cluster machine learning algorithms and advanced network analyses reveal similar clusters. A comparison between the results of the current study and the aforementioned studies shows some overlap in the identified clusters. The study using PMRF found six main clusters: psychological symptoms, hormonal symptoms, respiratory symptoms, nutritional symptoms, CTXrelated symptoms, and pain and abdominal symptoms [24]. Particularly noteworthy is the separation between psychological and physical symptoms. The study using concordance networks found three major clusters:
Fatigue/Constipation, Fatigue/Sleep, and Fatigue/Sleep/ Neck Spine [25]; where the only overlap with the current results can be found in the Fatigue/Sleep cluster.

Whether this is due to the analytical method, the measurement method of symptoms, differences in population, or other factors is impossible to determine at this time. It would be of great interest for future research to investigate how resulting clusters differ between various machine learning clustering algorithms (e.g. HDBSCAN, DBSCAN, GMM), various network analyses (e.g. PMRF, concordance networks), and various more traditional clustering analyses (e.g. EFA, CFA). Research focused on comparing analytical methodology can use data simulation methods to validate which methods reproduce the most accurate clusters as the investigators directly control the existence of clusters. A data simulation approach can be supplemented with a comparison of results from real data to provide a holistic view of the results of differing clustering methods. Such a comparison can inform the previously identified objective of identifying robust "new analytic strategies for symptom cluster research" by the 2015 expert panel [10].

One strength, but simultaneously a limitation, of this study is the use of different measurement tools for each separate symptom and health determinant. It has been argued that a standardization of how to measure symptoms for use in classifying symptom clusters is necessary for reproducible and valid interpretations [10]. However, the use of multiple (standardized and validated) measurement instruments creates the possibility to analyse many more symptoms and health determinants than would be possible when using only one standardized measurement tool.

There are three further limitations in regards to the way data was analyzed. First, while HDBSCAN was particularly suited for the current research question; due to the explorative nature of the analysis there is a distinct lack of easily interpretable fit metrics $[12,20]$. While the proportion of noise provides us with information on the amount of data points that could not be allocated to a cluster, it is unclear when the amount of noise is too high. Second, by focusing on the extraction of smaller clusters we found a seemingly high noise count in the cluster analyses. This indicates that there may be other (likely larger) clusters that could be extracted with other parameter settings. The amount of noise data points could also likely be reduced by using an algorithm that searches for the optimal minimum cluster size (i.e. hyperparameter tuning). While such settings were judged not optimal to answer our particular research question, such analyses could improve the fit of the model to the data. Third, for users that filled in Oncokompas more than once, we chose a random data row to ensure that we did not increase bias in our data set. 
Another dataset (e.g. a non-random selection of the data row with most moderate-to-high risk scores) could have produced a different result.

The research line of symptom clusters may eventually lead to practical applications in patient care. Currently we find ourselves in the explorative phase where plausible clusters need to be identified through the use of robust explorative analysis techniques. To move towards a confirmatory phase of research, the identified symptom and health determinant clusters need to be replicated in samples using other measurement techniques as well as using confirmatory analysis techniques. Stable and replicable symptom clusters need to be identified. As such, it is of interest to see whether the symptom and health determinant cluster found in the current study can be replicated in other research.. A form of standardization on both measurement techniques and (confirmatory) analysis techniques has been argued [10], but which measurement and analysis techniques are most appropriate and should be the standard has not yet been firmly concluded. Through replication, information on comparability can be gained.

To move beyond the confirmatory phase, and towards a phase of practical implementation, causal modelling techniques could be used to investigate possible etiological connections between symptoms, as well as by using subgroup analyses for differing tumor types. In particular, an investigation of how these clusters may change over time is of interest for such causal analyses. Furthermore, implementation of risk and protective factors in future models can help identify which symptoms may be best targeted as well as how care for individual patients should be tailored [26].

Knowledge regarding symptom clusters may inform targeted interventions [10]. While the current study cannot attest to etiology or causality within the found symptom and health determinants cluster, the main finding of interest for clinicians is the association between physical symptoms and psycho-social symptoms and health determinants for patients facing severe symptoms and health determinant issues. As such, it is advisable to assess whether a patient may profit from psycho-social support when suffering from (multiple) severe physical symptoms, in addition to treatment of the physical symptoms themselves.

\footnotetext{
Authors' contributions

K.I.N., C.F.W.P., P.C., and I.V.dL. planned the study. K.I.N. wrote the main manuscript text, prepared the figures, and carried out all analyses. C.F.W.P. consulted and checked the analyses. All authors reviewed the manuscript. The author(s) read and approved the final manuscript.
}

\section{Funding}

This study was funded by the Dutch Cancer Society, grant number VUP 2014-7202.

\section{Availability of data and materials}

The datasets generated and/or analyzed during the current study are not publicly available due to the sensitivity of health data and due to the fact that during the informed consent procedure participants did not consent to the publishing of generated data. Data are available from the corresponding author on reasonable request.

\section{Declarations}

Ethics approval and consent to participate

Part of the study population participated in various studies investigating feasibility [15] and effectiveness [13, 14] of Oncokompas. Ethical approval and informed consent procedures are reported in the relevant publications. The remaining part of the study population used Oncokompas as part of routine cancer care. Users provide consent for their data to be used in research during their account registration. Usage of data derived from Oncokompas is according to Dutch and European laws and regulations. No administrative permissions were required as data was not collected from medical records.

\section{Competing interests}

Carel F.W. Peeters is a member of the Editorial Board of BMC Medical Research Methodology.

\section{Author details}

${ }^{1}$ Department of Clinical, Vrije Universiteit Amsterdam, Neuro- and Developmental Psychology, Amsterdam Public Health Research Institute, Van der Boechorststraat 1, 1081, BT, Amsterdam, The Netherlands. ${ }^{2}$ Amsterdam UMC, Cancer Center Amsterdam, Amsterdam, The Netherlands. ${ }^{3}$ Department of Epidemiology \& Biostatistics, Amsterdam UMC, location VUmc, Boelelaan, 1117 Amsterdam, The Netherlands. ${ }^{4}$ Mathematical \& Statistical Methods Group (Biometris), Wageningen University \& Research, Wageningen, The Netherlands. ${ }^{5}$ Department of General Practice, Amsterdam UMC, location AMC, Amsterdam Public Health, Meibergdreef 9, Amsterdam, The Netherlands. ${ }^{6}$ Department of Otolaryngology-Head and Neck Surgery, Amsterdam UMC, location VUmc, Boelelaan, 1117 Amsterdam, The Netherlands.

Received: 13 November 2020 Accepted: 21 July 2021

Published online: 16 August 2021

\section{References}

1. Chen M-L, Lin C-C. Cancer symptom clusters: a validation study. J Pain Symptom Manag. 2007;34(6):590-9. https://doi.org/10.1016/J.JPAINSYMMA N.2007.01.008.

2. Sanson-Fisher R, Girgis A, Boyes A, Bonevski B, Burton L, Cook P, et al. The unmet supportive care needs of patients with cancer. Cancer. 2000:88:22637. https://doi.org/10.1002/(SICI)1097-0142(20000101)88:1<226::AID-CNCR3 $0>3.0 . \mathrm{CO} ; 2-\mathrm{P}$

3. Aaronson NK, Mattioli V, Minton O, Weis J, Johansen C, Dalton SO, et al. Beyond treatment - psychosocial and behavioural issues in cancer survivorship research and practice. Eur J Cancer Suppl. 2014;12(1):54-64 https://doi.org/10.1016/J.EJCSUP.2014.03.005.

4. Jansen F, van Uden-Kraan CF, van Zwieten V, Witte BI, Verdonck-de Leeuw IM. Cancer survivors' perceived need for supportive care and their attitude towards self-management and eHealth. Support Care Cancer. 2015;23(6): 1679-88. https://doi.org/10.1007/s00520-014-2514-7.

5. Irwin MR, Olmstead RE, Ganz PA, Haque R. Sleep disturbance, inflammation and depression risk in cancer survivors. Brain Behav Immun. 2013;30:S58-67. https://doi.org/10.1016/J.BBI.2012.05.002.

6. Cuijpers P, Beekman A, Smit F, Deeg D. Predicting the onset of major depressive disorder and dysthymia in older adults with subthreshold depression: a community based study. Int J Geriatr Psychiatry. 2006;21(9): 811-8. https://doi.org/10.1002/gps.1565.

7. Pullens MJJ, De Vries J, Roukema JA. Subjective cognitive dysfunction in breast cancer patients: a systematic review. Psycho-Oncology. 2010;19(11): 1127-38. https://doi.org/10.1002/pon.1673.

8. Alfano C, Rowland J. Recovery issues in cancer survivorship: a new challenge for supportive care. Cancer J. 2006;12(5):432-43. https://doi.org/1 0.1097/00130404-200609000-00012. 
9. Kirkova J, Aktas A, Walsh D, Davis MP. Cancer symptom clusters: clinical and research methodology. J Palliat Med. 2011;14(10):1149-66. https://doi.org/1 0.1089/.jpm.2010.0507.

10. Miaskowski C, Barsevick A, Berger A, Casagrande R, Grady PA, Jacobsen P, et al. Advancing symptom science through symptom cluster research: expert panel proceedings and recommendations. J Natl Cancer Inst. 2017; 109(4):djw253. https://doi.org/10.1093/jnci/djw253.

11. Ward Sullivan $C$, Leutwyler H, Dunn LB, Miaskowski C. A review of the literature on symptom clusters in studies that included oncology patients receiving primary or adjuvant chemotherapy. J Clin Nurs. 2018;27(3-4):51645. https://doi.org/10.1111/jocn.14057.

12. Campello RJGB, Moulavi D, Sander J. Density-based clustering based on hierarchical density estimates. Berlin: Springer; 2013. p. 160-72.

13. van der Hout $A$, van Uden-Kraan CF, Witte Bl, Coupé VMH, Jansen F, Leemans CR, et al. Efficacy, cost-utility and reach of an eHealth selfmanagement application 'Oncokompas' that helps cancer survivors to obtain optimal supportive care: study protocol for a randomised controlled trial. Trials. 2017;18(1):228. https://doi.org/10.1186/s13063-017-1952-1.

14. Duineveld LAM, Wieldraaijer T, van Asselt KM, Nugteren IC, Donkervoort SC, van de Ven $\mathrm{AWH}$, et al. Improving care after colon cancer treatment in the Netherlands, personalised care to enhance quality of life (I CARE study): study protocol for a randomised controlled trial. Trials. 2015;16(1):284. https://doi.org/10.1186/s13063-015-0798-7.

15. Melissant HC, Verdonck-de Leeuw IM, Lissenberg-Witte BI, Konings IR, Cuijpers P, van Uden-Kraan CF. 'Oncokompas', a web-based selfmanagement application to support patient activation and optimal supportive care: a feasibility study among breast cancer survivors. Acta Oncol. 2018;57(7):924-34. https://doi.org/10.1080/0284186X.2018.1438654.

16. van Leeuwen $\mathrm{M}$, Husson $\mathrm{O}$, Alberti $\mathrm{P}$, et al. Understanding the quality of life (QOL) issues in survivors of cancer: towards the development of an EORTC QOL cancer survivorship questionnaire. Health Qual Life Outcomes. 2018; 16(1):114. https://doi.org/10.1186/s12955-018-0920-0.

17. Lubberding S, van Uden-Kraan CF, Te Velde EA, et al. Improving access to supportive cancer care through an eHealth application: a qualitative needs assessment among cancer survivors. J Clin Nurs. 2015;24(9-10):1367-79. https://doi.org/10.1111/jocn.12753.

18. R Core Team. R: a language and environment for statistical computing Vienna: R Foundation for Statistical Computing; 2017.

19. Python Software Foundation. Python Language Reference, version 3.7.1. Available at http://www.python.org.

20. Mcinnes L, Healy J, Astels S. Hdbscan: hierarchical density based clustering. J Open Soft. 2017;2(11). https://doi.org/10.21105/joss.00205.

21. Ester M, Kriegel H, Sander J, Xu X. A density-based algorithm for discovering clusters in large spatial databases with noise. In: Proceedings of the second international conference on knowledge discovery and data mining (kdd-96): AAAI Press; 1996.

22. Pedersen TL. Tidygraph: a tidy api for graph manipulation; 2018.

23. Pedersen TL. Ggraph: an implementation of grammar of graphics for graphs and networks; 2018.

24. Papachristou N, Barnaghi P, Cooper B, Kober KM, Maguire R, Paul SM, et al. Network analysis of the multidimensional symptom experience of oncology. Sci Rep. 2019;9(1):2258. https://doi.org/10.1038/s41598-018-36973-1.

25. Henry TR, Marshall SA, Avis NE, Levine BJ, Ip EH. Concordance networks and application to clustering cancer symptomology. PLoS One. 2018;13(3): e0191981. https://doi.org/10.1371/journal.pone.0191981.

26. Schellekens MPJ, Wolvers MDJ, Schroevers MJ, Bootsma TI, Cramer AOJ, van der Lee ML. Exploring the interconnectedness of fatigue, depression, anxiety and potential risk and protective factors in cancer patients: a network approach. J Behav Med. 2019;43(4):553-63. https://doi.org/10.1007/ s10865-019-00084-7.

\section{Publisher's Note}

Springer Nature remains neutral with regard to jurisdictional claims in published maps and institutional affiliations.

Ready to submit your research? Choose BMC and benefit from:

- fast, convenient online submission

- thorough peer review by experienced researchers in your field

- rapid publication on acceptance

- support for research data, including large and complex data types

- gold Open Access which fosters wider collaboration and increased citations

- maximum visibility for your research: over $100 \mathrm{M}$ website views per year

At BMC, research is always in progress.

Learn more biomedcentral.com/submissions 